\title{
Geometric Process-Based Maintenance and Optimization Strategy for the Energy Storage Batteries
}

\author{
Yan Li, Peng Han, Jinkuan Wang, and Xin Song \\ School of Computer Science and Engineering, Northeastern University, Shenyang 110819, China \\ Correspondence should be addressed to Peng Han; hp@mail.neuq.edu.cn
}

Received 1 February 2016; Accepted 2 March 2016

Academic Editor: Jurgita Antucheviciene

Copyright ( 2016 Yan Li et al. This is an open access article distributed under the Creative Commons Attribution License, which permits unrestricted use, distribution, and reproduction in any medium, provided the original work is properly cited.

\begin{abstract}
Renewable energy is critical for improving energy structure and reducing environment pollution. But its strong fluctuation and randomness have a serious effect on the stability of the microgrid without the coordination of the energy storage batteries. The main factors that influence the development of the energy storage system are the lack of valid operation and maintenance management as well as the cost control. By analyzing the typical characteristics of the energy storage batteries in their life cycle, the geometric process-based model including the deteriorating system and the improving system is firstly built for describing the operation process, the preventive maintenance process, and the corrective maintenance process. In addition, this paper proposes an optimized management strategy, which aims to minimize the long-run average cost of the energy storage batteries by defining the time interval of the detection and preventive maintenance process as well as the optimal corrective maintenance times, subjected to the state of health and the reliability conditions. The simulation is taken under the built model by applying the proposed energy storage batteries' optimized management strategy, which verifies the effectiveness and applicability of the management strategy, denoting its obvious practicality on the current application.
\end{abstract}

\section{Introduction}

Along with the quality of environment being more and more emphasized, the microgrid, which regards the renewable resources as the major energy source, is growing rapidly in recent years. Energy storage technology in the microgrid system indicates tremendous development potential since it could solve the fluctuation and randomness problems of the renewable energy generation to a great extent $[1,2]$. It achieves a smooth output of the power by effectively regulating the voltage, frequency, or phase changes so that the largescale wind power and photovoltaic power generation will be incorporated into the microgrid reliably [3].

Main energy storage devices in the microgrid include the energy storage batteries, energy storage inductors, and energy storage capacitors, where the energy storage batteries are mostly used. Aimed at achieving integrated scheduling for regulating both the generation side and distribution side, all of the energy storage batteries are installed centrally in the charging room, considering the energy storage batteries' deteriorating characteristics with aging as well as the degradation of performance during times of charging and discharging. Strict maintenance and management aimed at the energy storage batteries are necessary for improving the electric energy efficiency, prolonging the service life, and reducing the life cycle costs $[4,5]$.

Munoz-Condes et al. [6] take into account the battery operating conditions and establish the mathematical relation between the impedance of simultaneous cells and the temperature for any model of lead-acid battery. Ure et al. [7] present a development and hardware implementation of an autonomous battery maintenance mechatronic system for the unmanned aerial vehicles. Li et al. [8] develop a multistep-ahead prediction model to predict the state of health and remaining life of the battery based on the mean entropy and relevance vector machine. Ablay [9] proposes a robust nonlinear estimator-based online condition monitoring method to determine the state of health of the battery systems online in the industry. Zhao et al. [10] develop an optimization model considering the lifetime characteristics of lead-acid batteries to realize the economic operation of a standalone microgrid via the nondominated sorting 


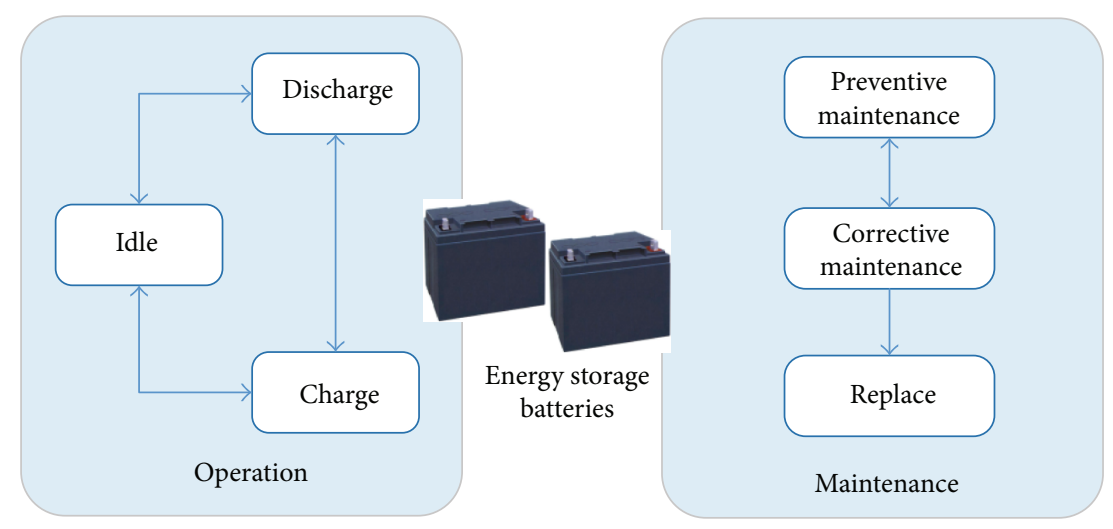

FIGURE 1: The states of operation and maintenance.

genetic algorithm. Liao and Köttig [11] focus on the hybrid prognostics approaches and leverage the advantages of the combining prognostics models for the battery's remaining useful life prediction. Han et al. [12] propose a novel health prediction model of the battery based on sample entropy and establish the prediction model by calculating the sample entropy. Wang and Liu [13] estimate the battery state of charge and implement the fault diagnosis according to the battery status to achieve the full and efficient use of battery power.

However, the previous estimation and monitoring studies related to the battery management mainly focus on the battery's prediction and monitoring but seldom distinguish and analyze the impact on the different states in the battery's life cycle including the operation, preventive maintenance, and corrective maintenance. And the geometric processbased (GP-based) method, with its practical advantages in characterizing the whole life cycle of component or system [14], gains large attention in recent years, and many researches have been taken based on GP in different application domains. Lam [15] introduces a geometric process $\delta$-shock maintenance model and adopts a replacement policy for minimizing the long-run average cost per unit time. Zhang [16] proposes a GP-based preventive repairment policy to solve the efficiency for a deteriorating and valuable system to minimize the average cost rate. Wang and Zhang [17] apply a bivariate preventive repair policy to solve the efficiency for a deteriorating and valuable system to minimize the average cost rate. Huang et al. [18] propose a delayed geometric process for the degenerative repairable systems by supposing the system to be returned to the "as good as former" state and the "worse" state with two different certain probabilities. Considering the application of the GP-based method on the energy storage batteries, the aging with the repeating charging and discharging, and the effect on the operation, preventive maintenance or corrective maintenance should be taken into account. Aimed at solving the energy storage batteries' optimized management, a GP-based model with the deteriorating system and the improving system of the energy storage batteries is firstly built, and then the long-run average cost of the energy storage batteries is optimized under the constraint of the detection or preventive maintenance time interval as well as the reliability demand through the proposed optimized management strategy.

\section{Typical State of the Energy Storage Battery}

Aimed at the daily behaviors of the energy storage batteries, the states of operation and maintenance are divided to distinguish the operation and maintaining processes, as is described in Figure 1.

In the operation process, the energy storage batteries are scheduled to charge if the excess renewable generated energy exists and discharge if the energy is deficient; otherwise, the energy storage batteries are on idle state. In the maintenance process, preventive maintenance is performed in certain time intervals depending on the defined function to enhance the energy storage batteries' performance and reduce the frequency of breakdown. The corrective maintenance is performed when the energy storage batteries break down or an obvious fault has occurred. After a period of time, the energy storage batteries would be replaced for some secondary applications when a certain index is unsatisfied.

Considering the aging of the energy storage batteries during the life cycle, the performance comparison analysis under the preventive maintenance state and corrective maintenance state are shown in Figure 2. The preventive maintenance process is helpful to improve the performance of the energy storage batteries to a certain extent, but the descending overall trend is indubitable for their chemical properties. The corrective maintenance process makes the disabled ones to work again, which avoids the premature discard of the energy storage batteries. Each of the maintenance processes is absolutely helpful and necessary at the exact time so that the appropriate maintenance strategy should be established based on the service condition.

\section{Mathematical Model of the Energy Storage Batteries}

The studied life cycle of the energy storage batteries starts from the first use and ends with the replacement, or the time interval between two contiguous replacements. Under the fact that the chemical property of the energy storage batteries is irreversible and degraded, the GP-based mathematical model of the studied life cycle is firstly proposed aimed at the typical processes, which includes the operation process, the detecting process, the preventive maintenance process, the 


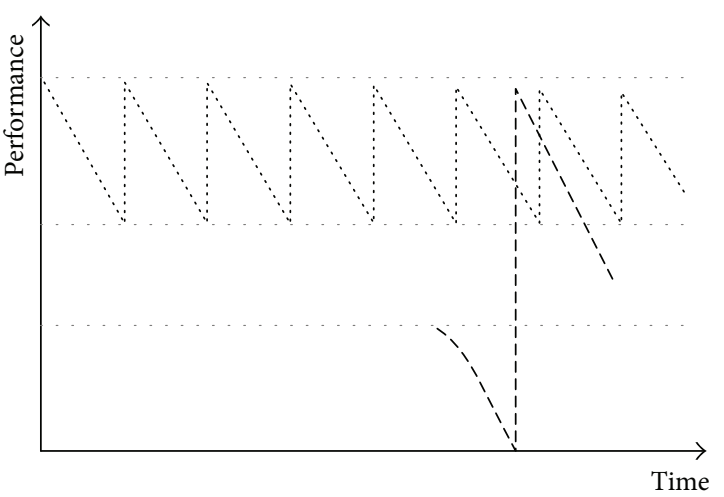

..... Preventive maintenance

--- Corrective maintenance

FIGURE 2: The comparison analysis under the two maintenance states.

corrective maintenance process, and so on [19]. The whole life cycle will be described in Figure 3.

For $n=1,2, \ldots, N$, the time interval between the $(n-1)$ th and the $n$th corrective maintenance in the life cycle can be defined as the $n$th period. Let $\left\{X_{n}^{i}, n=1,2, \ldots, i=1,2, \ldots\right\}$ be the $i$ th operation time of the energy storage batteries after $i$ - 1th preventive maintenance in the $n$th period. Let $\left\{Y_{n}^{i}, n=\right.$ $1,2, \ldots, i=1,2, \ldots\}$ be the $i$ th preventive maintenance in the $n$th period. Given that each preventive maintenance is accompanied by a detecting process, the detections have the same number of the preventive maintenances $\left\{M_{n}, n=\right.$ $1,2, \ldots, N\}$ in the $n$th period. Besides, the corrective maintenance time in the $n$th period is $\left\{Z_{n}, n=1,2, \ldots\right\}$, and the replacement time is $H$.

Now, the definitions and assumptions are made as follows to establish the related mathematical model of the energy storage batteries.

Definition 1. The successive operation time $\left\{X_{n}^{i}, i=1,2, \ldots\right\}$ in the $n$th period forms a GP with ratio $a$ and $E\left(X_{1}^{1}\right)=\lambda$, and the preventive maintenance time $\left\{Y_{n}^{i}, i=1,2, \ldots\right\}$ in the $n$th period forms a GP with ratio $b$ and $E\left(Y_{1}^{1}\right)=\mu$, while the consecutive corrective maintenance time $\left\{Z_{n}, n=1,2, \ldots\right\}$ constitutes a GP with radio $c$ and $E\left(Z_{1}\right)=\nu$. Besides, the replacement time $H$ is a random variable with $E(H)=\tau$.

Definition 2. The distribution function and probability density function of $X_{n}^{i}$ are $F\left(a^{n-1} x\right)$ and $a^{n-1} f\left(a^{n-1} x\right)$, respectively.

Definition 3. The preventive and corrective maintenance cost rates are, respectively, $c_{\mathrm{p}}$ and $c_{\mathrm{c}}$, the benefit rate of the operation is $c_{\mathrm{b}}$, the detection cost rate is $c_{\mathrm{d}}$, the replacement cost rate is $c_{\mathrm{r}}$ corresponding to the replacement time $H$, the unit price of a new energy storage battery is $c_{\mathrm{N}}$, and the secondary applications benefit of the depleted one is $c_{s}$.

Assumption 4. $\left\{X_{n}^{i}, i=1,2, \ldots\right\},\left\{Y_{n}^{i}, i=1,2, \ldots\right\}$, and $\left\{Z_{n}, n=1,2, \ldots\right\}$ are independent. In addition, the processes
$X_{n}^{i}, i=1,2, \ldots, M_{n}+1, n=1,2, \ldots, N$ and $Y_{n}^{i}, i=$ $1,2, \ldots, M_{n}, n=1,2, \ldots, N$ are independent and identically distributed (i.i.d.) random variables.

Assumption 5. The operation time decreases with age; thus it is a stochastically decreasing GP-based model with $a>1$. And the preventive maintenance and corrective maintenance processes are stochastically increasing GP-based model with $0<b<1$ and $0<c<1$.

\section{GP-Based Optimized Management Strategy}

Economy and reliability are the focus of most concern for the construction of microgrid and even the energy storage system. The evaluation aimed at the economy of the energy storage system will be expressed as the average cost of the energy storage batteries. Generally, the average cost is given by the ratio of the total cost and the life cycle, as is shown in function (1):

$$
C(T)=\lim _{t \rightarrow \infty} \frac{C(t)}{t} .
$$

Depending on the definitions of the energy storage batteries above, the successive periods form a renewal process; therefore, the successive periods together with the cost rates incurred in each period constitute a renewal process. Supposing an optimized management strategy $(T, N)$ is applied, the long-run average cost $C(T, N)$ that is expressed by the defined GP-based processes is given by

$$
C(T, N)=\frac{C(N)}{T_{\text {Total }}},
$$

where

$$
\begin{aligned}
& C(N)=E\left(c_{\mathrm{d}} \sum_{n=1}^{N} M_{n}+c_{\mathrm{p}} \sum_{n=1}^{N} \sum_{i=1}^{M_{n}} Y_{n}^{i}+c_{\mathrm{c}} \sum_{n=1}^{N-1} Z_{n}+c_{\mathrm{r}} H\right. \\
& \left.+c_{\mathrm{N}}-c_{\mathrm{s}}-c_{\mathrm{b}} \sum_{n=1}^{N} X_{n}\right), \\
& T_{\text {Total }}=E\left(\sum_{n=1}^{N} X_{n}+\sum_{n=1}^{N} \sum_{i=1}^{M_{n}} Y_{n}^{i}+\sum_{n=1}^{N-1} Z_{n}+H\right) .
\end{aligned}
$$

Then, from function (2)-(3) the long-run average cost of the energy storage batteries is shown as function (4):

$$
\begin{gathered}
C(T, N)=E\left(c_{\mathrm{d}} \sum_{n=1}^{N} M_{n}+c_{\mathrm{p}} \sum_{n=1}^{N} \sum_{i=1}^{M_{n}} Y_{n}^{i}+c_{\mathrm{c}} \sum_{n=1}^{N-1} Z_{n}\right. \\
\left.+c_{\mathrm{r}} H+c_{\mathrm{N}}-c_{\mathrm{s}}-c_{\mathrm{b}} \sum_{n=1}^{N} X_{n}\right) \\
\cdot\left(E\left(\sum_{n=1}^{N} X_{n}+\sum_{n=1}^{N} \sum_{i=1}^{M_{n}} Y_{n}^{i}+\sum_{n=1}^{N-1} Z_{n}+H\right)\right)^{-1}
\end{gathered}
$$




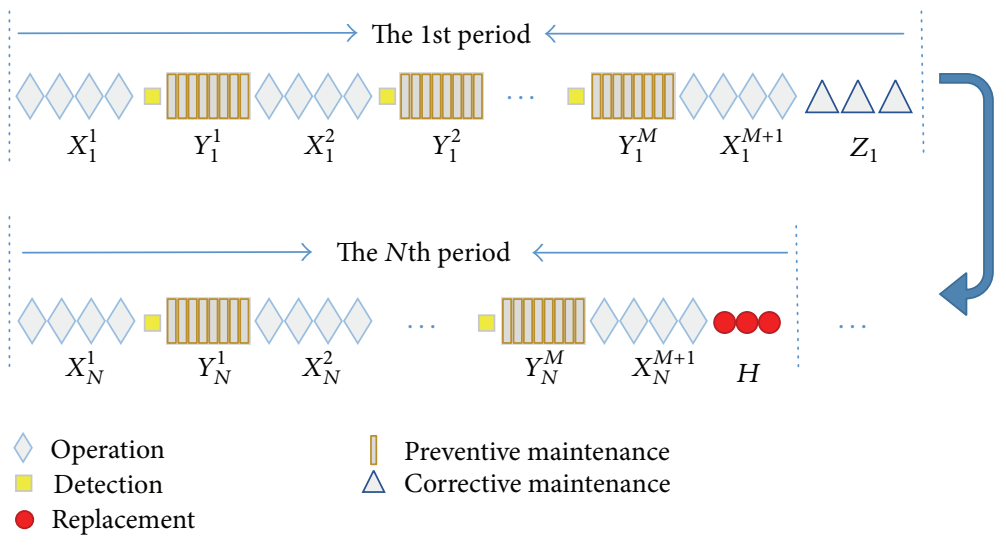

FIGURE 3: The whole life cycle of the energy storage batteries.

$$
\begin{aligned}
& =\left(c_{\mathrm{d}} \sum_{n=1}^{N} E\left(M_{n}\right)+c_{\mathrm{p}} \sum_{n=1}^{N} \frac{\mu}{b^{n-1}} E\left(M_{n}\right)+c_{\mathrm{c}} \sum_{n=1}^{N-1} \frac{\nu}{c^{n-1}}\right. \\
& \left.+c_{\mathrm{r}} \tau+c_{\mathrm{N}}-c_{\mathrm{s}}-c_{\mathrm{b}} \sum_{n=1}^{N} E\left(X_{n}\right)\right)\left(\sum_{n=1}^{N} E\left(X_{n}\right)\right. \\
& \left.+\sum_{n=1}^{N} \frac{\mu}{b^{n-1}} E\left(M_{n}\right)+\sum_{n=1}^{N-1} \frac{v}{c^{n-1}}+\tau\right)^{-1} .
\end{aligned}
$$

To further obtain the explicit result of $C(T, N)$, the number of preventive maintenances $M_{n}$ is supposed to have a geometric distribution, that is $M_{n} \sim G\left(p_{n}\right)$, and then

$$
\begin{gathered}
P\left(X_{n}^{1} \leq T\right)=F\left(a^{n-1} T\right)=q_{n}^{i} p_{n}, \quad i=0,1,2,3, \ldots, \\
q_{n}=P\left(X_{n}^{1}>T\right)=1-p_{n} .
\end{gathered}
$$

Consequently,

$$
E\left(M_{n}\right)=\frac{q_{n}}{p_{n}}=\frac{1-p_{n}}{p_{n}} .
$$

Then, the detection time and preventive maintenance time in the life cycle are transformed into

$$
\begin{aligned}
E\left(\sum_{n=1}^{N} M_{n}\right) & =\sum_{n=1}^{N} \frac{1-p_{n}}{p_{n}} \\
E\left(\sum_{n=1}^{N} \sum_{i=1}^{M_{n}} Y_{n}^{i}\right) & =\sum_{n=1}^{N} \frac{\mu}{b^{n-1}} \frac{1-p_{n}}{p_{n}} .
\end{aligned}
$$

Let $E\left(X_{n}\right)=\psi(T, n)$ be the expected operation time in the $n$th period:

$$
\begin{aligned}
E\left(X_{n}\right) & =\psi(T, n) \\
& =E\left(\frac{1}{F\left(a^{n-1} T\right)} \int_{0}^{T}\left(a^{n-1} t\right) d F\left(a^{n-1} t\right)\right) \\
& =\frac{1}{F\left(a^{n-1} T\right)} \int_{0}^{T}\left(1-F\left(a^{n-1} t\right)\right) d t
\end{aligned}
$$

Given that the operation time has a Weibull distribution with density function,

$$
f(t)= \begin{cases}\frac{k}{\lambda}\left(\frac{t}{\lambda}\right)^{k-1} \exp \left(-\left(\frac{t}{\lambda}\right)^{k}\right), & t>0 \\ 0, & \text { else. }\end{cases}
$$

Then,

$$
\begin{aligned}
& p_{n}=F\left(a^{n-1} T\right)=1-\exp \left(-\left(\frac{1}{\lambda} a^{n-1} T\right)^{k}\right) \\
& q_{n}=1-p_{n}=\exp \left(-\left(\frac{1}{\lambda} a^{n-1} T\right)^{k}\right) \\
& \psi(T, n)=\frac{1}{1-\exp \left(-\left((1 / \lambda) a^{n-1} T\right)^{k}\right)} \\
& \cdot \int_{0}^{t} \exp \left(-\left(\frac{1}{\lambda} a^{n-1} t\right)^{k}\right) d t=\frac{\lambda}{a^{n-1}} .
\end{aligned}
$$

From function (4), (6), and (10)-(11), $C(N, T)$ will be further expressed as

$$
\begin{aligned}
& C(T, N)=\left(c_{\mathrm{d}} \sum_{n=1}^{N} \frac{\exp \left(-\left((1 / \lambda) a^{n-1} T\right)^{k}\right)}{1-\exp \left(-\left((1 / \lambda) a^{n-1} T\right)^{k}\right)}\right. \\
& +c_{\mathrm{p}} \sum_{n=1}^{N} \frac{\mu}{b^{n-1}} \frac{\exp \left(-\left((1 / \lambda) a^{n-1} T\right)^{k}\right)}{1-\exp \left(-\left((1 / \lambda) a^{n-1} T\right)^{k}\right)} \\
& \left.+c_{\mathrm{c}} \sum_{n=1}^{N-1} \frac{v}{c^{n-1}}+c_{\mathrm{r}} \tau+c_{\mathrm{N}}-c_{\mathrm{s}}-c_{\mathrm{b}} \sum_{n=1}^{N} \frac{\lambda}{a^{n-1}}\right)
\end{aligned}
$$




$$
\begin{aligned}
& \cdot\left(\sum_{n=1}^{N} \frac{\lambda}{a^{n-1}}+\sum_{n=1}^{N} \frac{\mu}{b^{n-1}} \frac{\exp \left(-\left((1 / \lambda) a^{n-1} T\right)^{k}\right)}{1-\exp \left(-\left((1 / \lambda) a^{n-1} T\right)^{k}\right)}\right. \\
& \left.+\sum_{n=1}^{N-1} \frac{v}{c^{n-1}}+\tau\right)^{-1} .
\end{aligned}
$$

Assuming that the preventive maintenance process would be taken before the state of health ( $\mathrm{SOH}$ ) is down to $R$, one has

$$
F\left(a^{n-1} T\right) \leq 1-R .
$$

From function (10), the constraint of $T$ has the following derivation:

$$
\begin{aligned}
1-\exp \left(-\left(\frac{1}{\lambda} a^{n-1} T\right)^{k}\right) & \leq 1-R, \\
\left(\frac{1}{\lambda} a^{n-1} T\right)^{k} & \leq \ln \left(\frac{1}{R}\right), \\
\frac{1}{\lambda} a^{n-1} T & \leq\left(\ln \left(\frac{1}{R}\right)\right)^{1 / k}, \\
T & \leq \frac{\lambda}{a^{n-1}}\left(\ln \left(\frac{1}{R}\right)\right)^{1 / k} .
\end{aligned}
$$

The reliability of the energy storage batteries is a representation of the full operation capability. Generally, it is defined as $A(T)=\lim _{t \rightarrow \infty}(A(t) / t)$. Under the proposed optimized management strategy aimed at the life cycle of the energy storage batteries, the reliability $A(N, T)$ will be expressed as the ratio of the operation time and the whole life cycle; that is,

$A(T, N)$

$$
=\frac{E\left(\sum_{n=1}^{N} X_{n}\right)}{E\left(\sum_{n=1}^{N} X_{n}+\sum_{n=1}^{N} \sum_{i=1}^{M_{n}} Y_{n}^{i}+\sum_{n=1}^{N-1} Z_{n}+H\right)} .
$$

Furtherly, from function (6), (8), (10)-(11), and (15),

$$
\begin{aligned}
A(T, N) & =\sum_{n=1}^{N} \frac{\lambda}{a^{n-1}}\left(\sum_{n=1}^{N} \frac{\lambda}{a^{n-1}}\right. \\
& \left.+\sum_{n=1}^{N} \frac{\mu}{b^{n-1}} \frac{\exp \left(-a^{n-1} T / \lambda\right)}{1-\exp \left(-a^{n-1} T / \lambda\right)}+\sum_{n=1}^{N-1} \frac{\nu}{c^{n-1}}+\tau\right)^{-1} .
\end{aligned}
$$

Based on the above analysis, the objective function of the proposed optimized management strategy is to find the minimum value of $C\left(T^{*}, N^{*}\right)$ against $T^{*}$ and $N^{*}$ under the constraint of the reliability threshold, which would be expressed as

$$
\begin{aligned}
C\left(T^{*}, N^{*}\right): \min \quad & C(T, N) \\
\text { s.t. } \quad & A\left(T^{*}, N^{*}\right) \geq A_{0}, \\
& T \leq \frac{\lambda}{a^{n-1}}\left(\ln \left(\frac{1}{R}\right)\right)^{1 / k},
\end{aligned}
$$

where $A_{0}$ is the threshold of the availability.

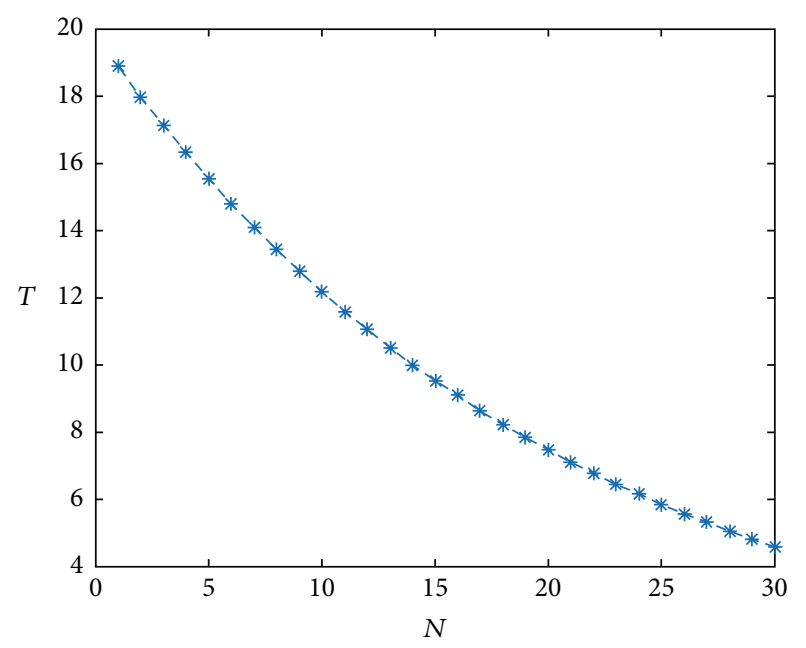

FIgURE 4: The constraint of $T$ against $N$.

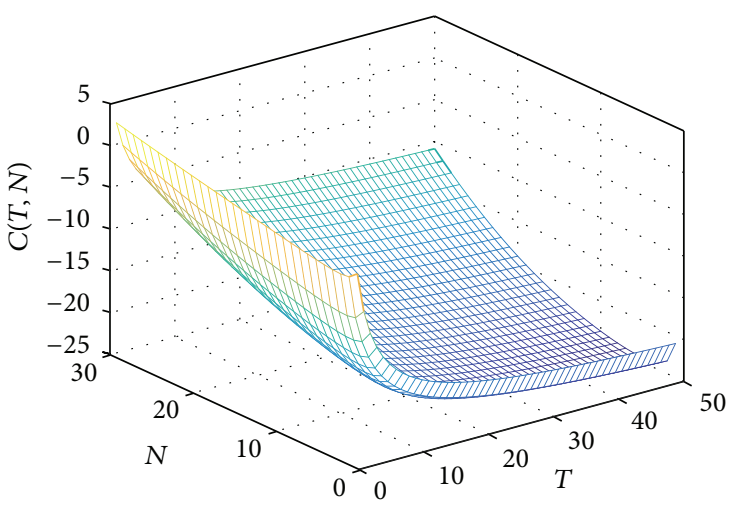

Figure 5: $C(T, N)$ against $T$ and $N$.

\section{Simulation Analysis}

To verify the proposed optimized management strategy of the energy storage batteries, numerical examples are analyzed as follows. Originally from the lead-acid storage battery, the main parameters are listed in Table 1 . Under the constraint of function (14), the time interval $T$ that determines the detection and preventive maintenance processes have a certain relation to the maximum number of corrective maintenances $N$, which satisfies the area under the curve, as is shown in Figure 4.

Considering the long-run average cost of the energy storage batteries $C(T, N)$ under the proposed optimized management strategy $(T, N)$, the simulation is firstly carried out to gain the minimum $C\left(T^{*}, N^{*}\right)$, and the result is illustrated in Figure 5. It indicates that the long-run average costs increase as the number of corrective maintenances goes up and decrease as the time interval of detection and preventive maintenance goes down. So the dense and multiple times of detection, preventive maintenance, and corrective maintenance would certainly raise the cost of the energy storage batteries. 
TABLE 1: Main parameters of the simulation.

\begin{tabular}{|c|c|c|c|c|c|c|c|c|c|c|c|c|c|c|c|c|}
\hline Parameter & $a$ & $b$ & $c$ & $\lambda$ & $\mu$ & $v$ & $\tau$ & $c_{\mathrm{b}}$ & $c_{\mathrm{d}}$ & $c_{\mathrm{p}}$ & $c_{\mathrm{c}}$ & $c_{\mathrm{r}}$ & $c_{\mathrm{N}}$ & $c_{\mathrm{s}}$ & $R$ & $k$ \\
\hline Value & 1.05 & 0.95 & 0.9 & 100 & 0.3 & 0.7 & 10 & 50 & 60 & 100 & 300 & 200 & 2000 & 200 & 0.8 & 0.9 \\
\hline
\end{tabular}

TABle 2: The main explicit values of $C(T, N)$.

\begin{tabular}{|c|c|c|c|c|c|c|c|c|c|}
\hline \multirow{2}{*}{$N$} & \multicolumn{9}{|c|}{$T$} \\
\hline & 7 & 9 & 10 & 11 & 12 & 14 & 16 & 17 & 18 \\
\hline 1 & -14.94 & -16.17 & -16.64 & -17.04 & -17.39 & -17.96 & -18.41 & -18.61 & -18.78 \\
\hline 2 & -16.88 & -18.23 & -18.74 & -19.18 & -19.57 & -20.19 & -20.69 & -20.90 & -21.09 \\
\hline 3 & -17.33 & -18.72 & -19.26 & -19.71 & -20.11 & -20.76 & -21.28 & $-21.50^{*}$ & -21.70 \\
\hline 4 & -17.41 & -18.83 & -19.37 & -19.84 & -20.24 & -20.91 & -21.44 & -21.67 & -21.87 \\
\hline 6 & -17.16 & -18.62 & -19.18 & -19.66 & -20.08 & -20.77 & -21.31 & -21.55 & -21.76 \\
\hline 9 & -16.42 & -17.91 & -18.48 & -18.97 & -19.40 & -20.11 & -20.67 & -20.91 & -21.13 \\
\hline 11 & -15.80 & -17.29 & -17.87 & -18.36 & -18.79 & -19.51 & -20.08 & -20.32 & -20.54 \\
\hline 13 & -15.10 & -16.59 & -17.16 & -17.66 & -18.09 & -18.81 & -19.38 & -19.62 & -19.85 \\
\hline 15 & -14.33 & -15.80 & -16.37 & -16.87 & -17.30 & -18.01 & -18.58 & -18.82 & -19.04 \\
\hline 19 & -12.57 & -13.98 & -14.53 & -15.00 & -15.41 & -16.10 & -16.65 & -16.88 & -17.09 \\
\hline
\end{tabular}

${ }^{*}$ The minimum long-run average cost of the energy storage batteries.

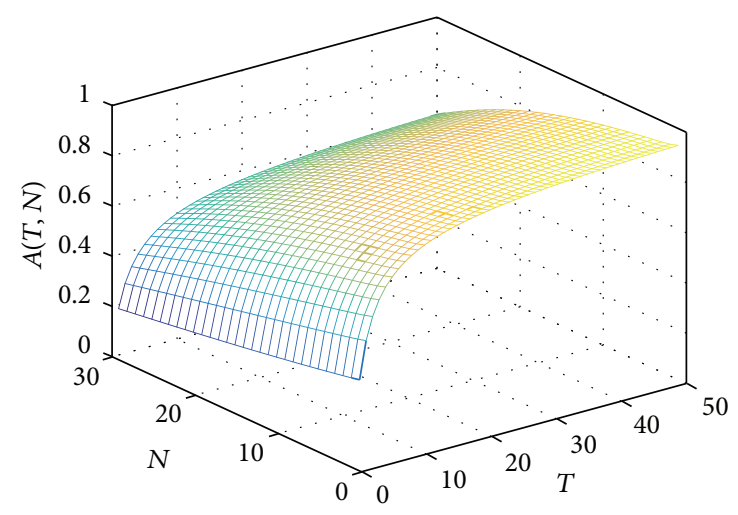

Figure 6: $A(T, N)$ against $T$ and $N$.

The main explicit values of the long-run average cost $C(T, N)$ are lists in Table 2.

The values that are formatted in the bold text are the constraint of $T$, the ones with the italic font are the invalid values. Comparing to the values which are accordant with the certain condition, the minimum long-run average cost of the energy storage batteries is $C(17,3)=-21.50$; that is, the lowest long-run average cost is gained when the time interval of detection and preventive maintenance is set to 17 and the maximum number of corrective maintenances is 3 .

The next simulation is taken to verify another constraint $A(T, N)$ of the proposed optimized management strategy. The threshold $A_{0}$ is set to 0.8 . Under the same conditions of the previous simulations, the reliability curve in the different values of $T$ and $N$ is shown in Figure 6, and the explicit values of $A(T, N)$ corresponding to Table 2 are listed in Table 3. Considering the optimized management strategy $(17,3)$, the corresponding reliability is 0.84 that meets the
0.8 threshold, which indicates a strong applicability of the proposed optimized management strategy.

\section{Conclusions}

Oriented to the centralized schedule and management of the energy storage batteries in the microgrid for dealing with the strong fluctuation and randomness of the renewable energy, this paper firstly analyzes the different main states in the life cycle of the energy storage batteries, which includes the operation state, the preventive maintenance state, and the corrective maintenance state. Considering the energy storage batteries' deteriorating characteristics during the repeating charging and discharging as well as the negative impact of aging, the deteriorating system and the improving system are distinguished in the built GP-based model for describing the operation process, preventive maintenance process, and corrective maintenance process. Moreover, this paper proposes the optimized management strategy to minimize the long-run average cost on the specified condition of the $\mathrm{SOH}$ and reliability, which adaptively schedules the time interval of the detection and preventive maintenance as well as the maximum number of corrective maintenances. Finally, the simulation with the specified $\mathrm{SOH}$ and reliability thresholds is taken to verify its effectiveness. The results denote that the built GP-based model is suitable to study the management of the energy storage batteries, and the proposed optimized management strategy will reduce the long-run average cost of the energy storage batteries on the promise of the normal operation, which is of much suitability for the energy storage batteries' optimized management.

\section{Competing Interests}

The authors declare that they have no competing interests. 
TABLE 3: The explicit values of $A(T, N)$.

\begin{tabular}{|c|c|c|c|c|c|c|c|c|c|}
\hline \multirow{2}{*}{$N$} & \multicolumn{9}{|c|}{$T$} \\
\hline & 7 & 9 & 10 & 11 & 12 & 14 & 16 & 17 & 18 \\
\hline 1 & 0.74 & 0.78 & 0.80 & 0.81 & 0.82 & 0.84 & 0.85 & 0.86 & 0.87 \\
\hline 2 & 0.73 & 0.77 & 0.79 & 0.80 & 0.81 & 0.83 & 0.84 & 0.85 & 0.85 \\
\hline 3 & 0.73 & 0.76 & 0.78 & 0.79 & 0.80 & 0.82 & 0.84 & $0.84^{*}$ & 0.85 \\
\hline 4 & 0.72 & 0.76 & 0.77 & 0.79 & 0.80 & 0.81 & 0.83 & 0.84 & 0.84 \\
\hline 6 & 0.70 & 0.74 & 0.76 & 0.77 & 0.78 & 0.80 & 0.82 & 0.82 & 0.83 \\
\hline 9 & 0.68 & 0.72 & 0.74 & 0.75 & 0.76 & 0.78 & 0.80 & 0.80 & 0.81 \\
\hline 11 & 0.66 & 0.70 & 0.72 & 0.73 & 0.75 & 0.77 & 0.78 & 0.79 & 0.79 \\
\hline 13 & 0.65 & 0.69 & 0.70 & 0.72 & 0.73 & 0.75 & 0.76 & 0.77 & 0.78 \\
\hline 15 & 0.63 & 0.67 & 0.69 & 0.70 & 0.71 & 0.73 & 0.75 & 0.75 & 0.76 \\
\hline 19 & 0.59 & 0.63 & 0.65 & 0.66 & 0.67 & 0.69 & 0.71 & 0.71 & 0.72 \\
\hline
\end{tabular}

${ }^{*}$ The reliability corresponding to $(17,3)$.

\section{Acknowledgments}

This work is supported by the National Natural Science Foundation of China (61374097 and 61473066), the Program for New Century Excellent Talents in University (NCET-120103), the Basic Science \& Research Fund of Northeastern University (N152303010) and of Northeastern University at Qinhuangdao (XNB201601), and the Science and Technology Research Project of Higher Education of Hebei Province (QN2016315).

\section{References}

[1] P. Han, J. Wang, Y. Han, and Y. Li, "Resident Plug-In Electric Vehicle charging modeling and scheduling mechanism in the smart grid," Mathematical Problems in Engineering, vol. 2014, Article ID 540624, 8 pages, 2014.

[2] D. Tran and A. M. Khambadkone, "Energy management for lifetime extension of energy storage system in micro-grid applications," IEEE Transactions on Smart Grid, vol. 4, no. 3, pp. 1289-1296, 2013.

[3] B. Liu, F. Zhuo, Y. Zhu, and H. Yi, "System operation and energy management of a renewable energy-based DC microgrid for high penetration depth application," IEEE Transactions on Smart Grid, vol. 6, no. 3, pp. 1147-1155, 2015.

[4] Y. Han, J. Wang, Q. Zhao, and P. Han, "An optimal operating strategy for battery life cycle costs in electric vehicles," Journal of Applied Mathematics, vol. 2014, Article ID 305905, 6 pages, 2014.

[5] H. Peng, W. Jinkuan, S. Xin, and L. Yan, "Geometric-processbased battery management modeling and optimization in the electric vehicle battery switching station," in Proceedings of the 11th International Conference on Natural Computation, pp. 897901, IEEE, Zhangjiajie, China, August 2015.

[6] P. Munoz-Condes, M. Gomez-Parra, C. Sancho et al., "On condition maintenance based on the impedance measurement for traction batteries: development and industrial implementation," IEEE Transactions on Industrial Electronics, vol. 60, no. 7, pp. 2750-2759, 2013.

[7] N. K. Ure, G. Chowdhary, T. Toksoz, J. P. How, M. A. Vavrina, and J. Vian, "An automated battery management system to enable persistent missions with multiple aerial vehicles," IEEE/
ASME Transactions on Mechatronics, vol. 20, no. 1, pp. 275-286, 2015.

[8] H. Li, D. Pan, and C. L. P. Chen, "Intelligent prognostics for battery health monitoring using the mean entropy and relevance vector machine," IEEE Transactions on Systems, Man, and Cybernetics: Systems, vol. 44, no. 7, pp. 851-862, 2014.

[9] G. Ablay, "Online condition monitoring of battery systems with a nonlinear estimator," IEEE Transactions on Energy Conversion, vol. 29, no. 1, pp. 232-239, 2014.

[10] B. Zhao, X. Zhang, J. Chen, C. Wang, and L. Guo, "Operation optimization of standalone microgrids considering lifetime characteristics of battery energy storage system," IEEE Transactions on Sustainable Energy, vol. 4, no. 4, pp. 934-943, 2013.

[11] L. Liao and F. Köttig, "Review of hybrid prognostics approaches for remaining useful life prediction of engineered systems, and an application to battery life prediction," IEEE Transactions on Reliability, vol. 63, no. 1, pp. 191-207, 2014.

[12] H. Han, H. Xu, Z. Yuan, and Y. Shen, "A new SOH prediction model for lithium-ion battery for electric vehicles," in Proceedings of the 17th International Conference on Electrical Machines and Systems (ICEMS '14), pp. 997-1002, Hangzhou, China, October 2014.

[13] Y. Wang and Y. Liu, "Electronic control system design and test of pure electric vehicle battery management system," in Proceedings of the 2nd International Conference on Mechanic Automation and Control Engineering (MACE '11), pp. 1289-1292, Hohhot, China, July 2011.

[14] Y. Lam and Y. L. Zhang, "A geometric-process maintenance model for a deteriorating system under a random environment," IEEE Transactions on Reliability, vol. 52, no. 1, pp. 83-89, 2003.

[15] Y. Lam, "A geometric process $\delta$-shock maintenance model," IEEE Transactions on Reliability, vol. 58, no. 2, pp. 389-396, 2009.

[16] Y. L. Zhang, "A geometric-process repair-model with good-asnew preventive repair," IEEE Transactions on Reliability, vol. 51, no. 2, pp. 223-228, 2002.

[17] G. J. Wang and Y. L. Zhang, "Optimal periodic preventive repair and replacement policy assuming geometric process repair," IEEE Transactions on Reliability, vol. 55, no. 1, pp. 118-122, 2006.

[18] A. L. Huang, Q. M. Li, M. Z. Ruan, and D. J. Mao, “Optimal replacement policies for degenerative repairable systems based 
on delayed geometric process," Journal of Systems Engineering and Electronics, vol. 33, no. 11, pp. 2449-2452, 2011.

[19] Y. Li, J.-K. Wang, P. Han, and Y.-H. Han, "Geometric-processbased battery management optimizing policy for the electric bus," Mathematical Problems in Engineering, vol. 2015, Article ID 624130, 7 pages, 2015. 


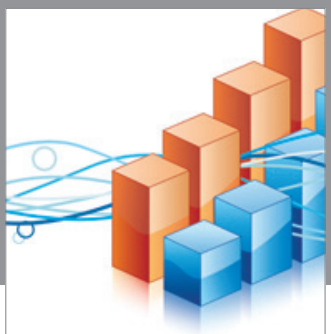

Advances in

Operations Research

vatem alat4

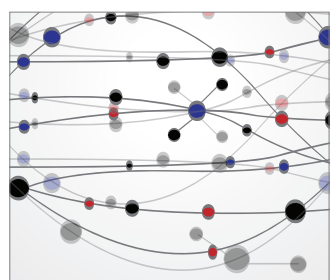

\section{The Scientific} World Journal
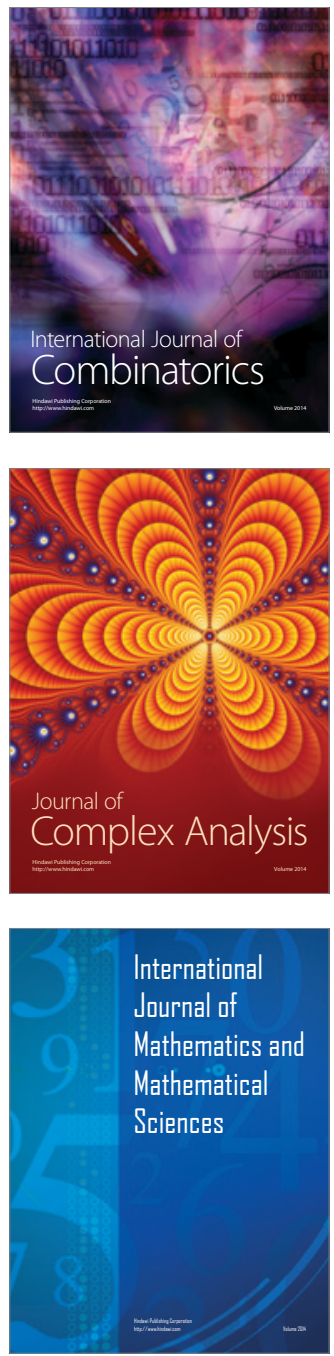
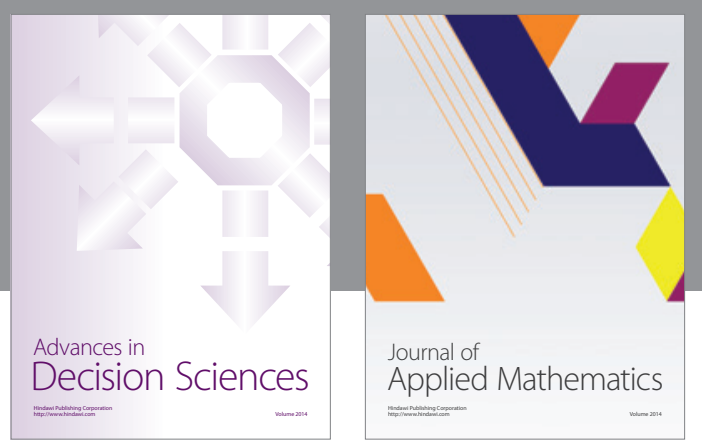

Algebra

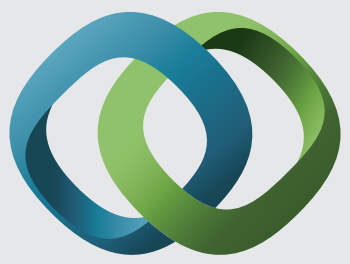

\section{Hindawi}

Submit your manuscripts at

http://www.hindawi.com
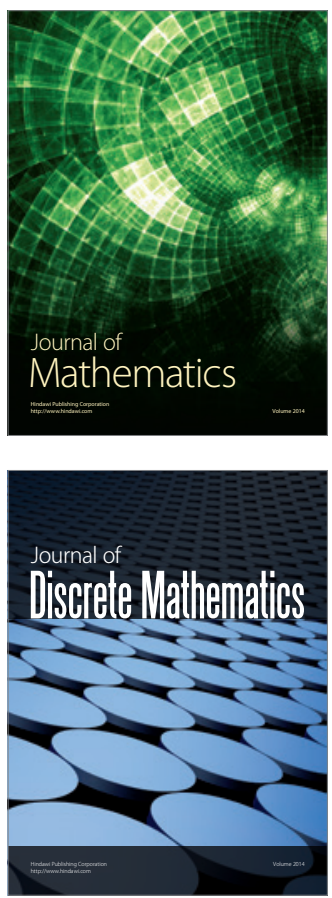

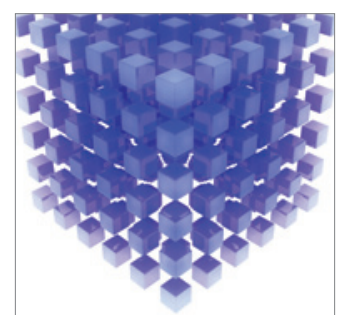

Mathematical Problems in Engineering
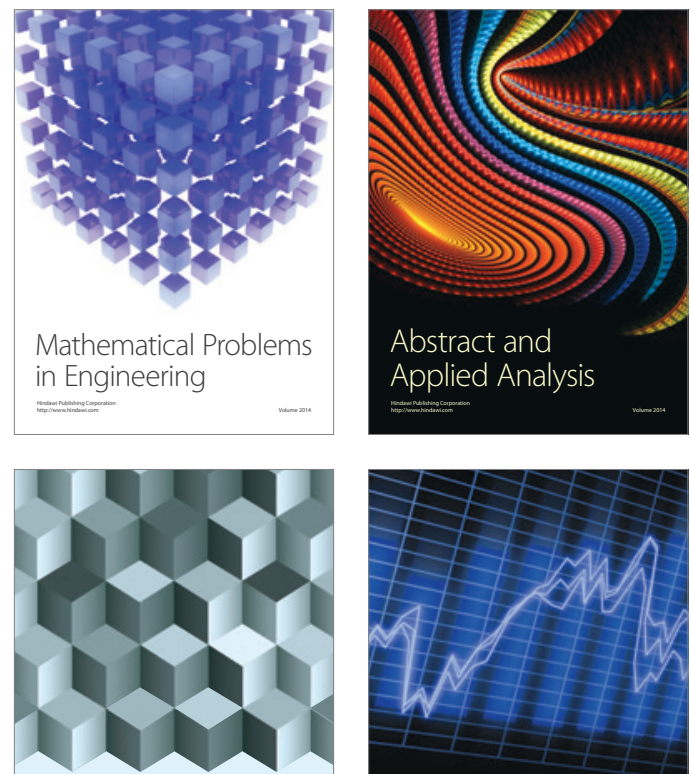

Journal of

Function Spaces

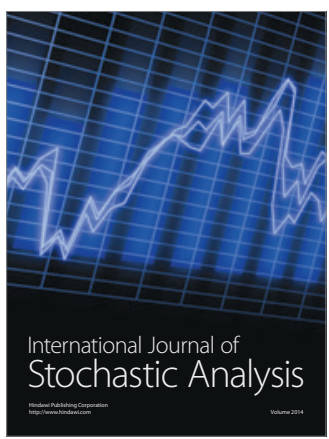

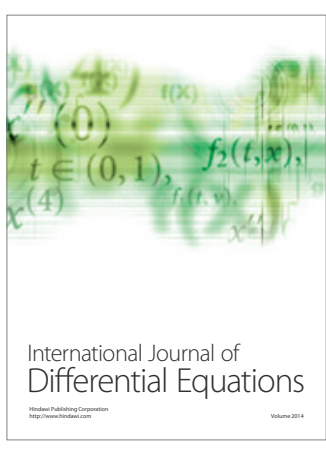
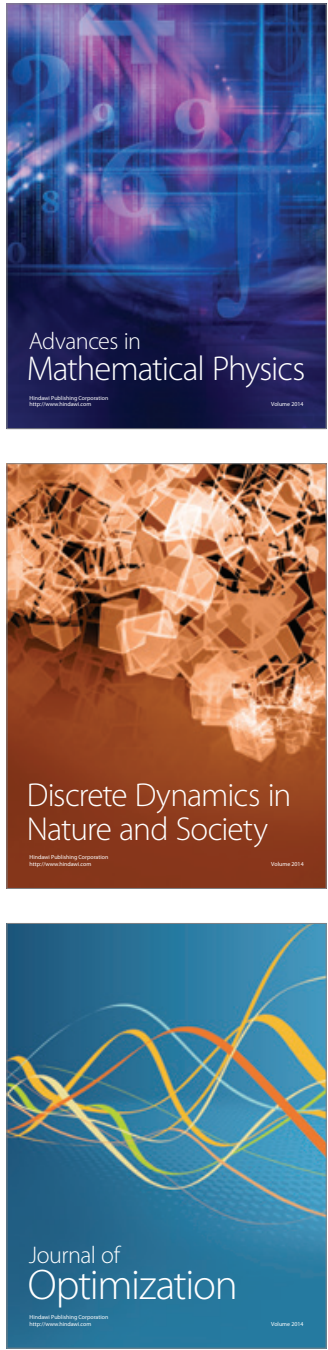